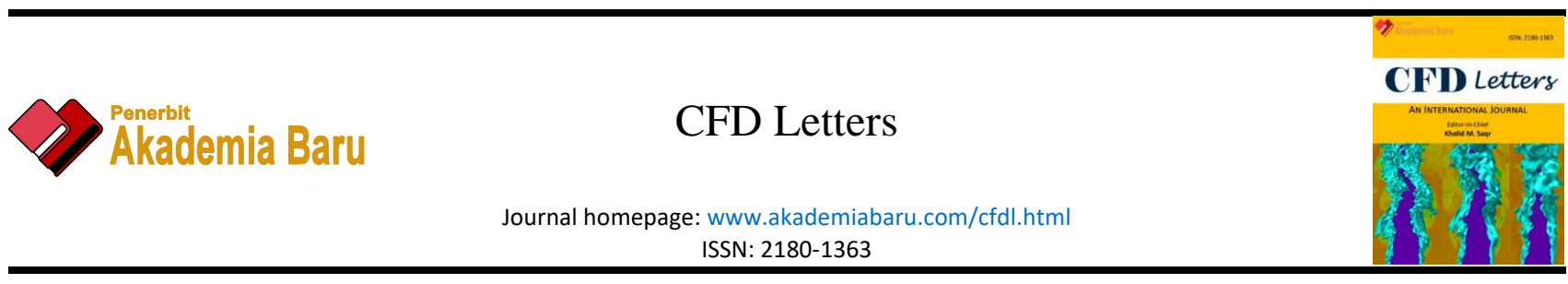

\title{
Numerical Computations of Biomagnetic Fluid Flow in a Lid Driven Cavity
}

\author{
Normazni Abdullah ${ }^{1}$, Zuhaila Ismail ${ }^{1,}{ }^{*}$, Adrian Syah Halifi ${ }^{1}$, Alia Rafiza Che Ayob ${ }^{1}$, Erwan Hafizi \\ Kasiman², Norsarahaida Saidina Amin ${ }^{1}$ \\ Department of Mathematical Sciences, Faculty of Science, Universiti Teknologi Malaysia, 81310 Skudai, Johor, Malaysia \\ Department of Water and Environmental Engineering, Faculty of Engineering, Universiti Teknologi Malaysia, 81310 Skudai, Johor, Malaysia
}

\section{ARTICLE INFO ABSTRACT}

\section{Article history:}

Received 19 February 2020 Received in revised form 16 April 2020 Accepted 21 April 2020

Available online 28 April 2020

\begin{abstract}
The study on the effects of biological fluids in the presence of magnetic field is known as biomagnetic fluid dynamics (BFD) and the most common biological fluid that exhibit such magnetic properties is blood. An extensive research work has been done in this area due to its applications in medical and bioengineering. Basically, it is essential for a study to be justified according to certain benchmark before progressing. Hence, biomagnetic fluid flow in a lid driven cavity is numerically investigated by utilizing two numerical schemes: finite difference and finite element methods. The formulation adopted is consistent with the principles of ferrohydrodynamics. The mathematical model describes Newtonian blood flow under the influence of a spatially varying magnetic field. The model considers the biofluid as non-conducting. The flow is assumed to be two-dimensional, steady, laminar and isothermal. The implementation of finite element method shows stability issue due to extremely steep magnetic field gradient while finite difference method shows no issue. Due to this, a solution is proposed to alleviate the problem and the result for various magnetic field intensity presented.
\end{abstract}

Keywords:

Biomagnetic Fluid Dynamics;

Finite Element Method;

Finite Difference Method

Copyright @ 2020 PENERBIT AKADEMIA BARU - All rights reserved

\section{Introduction}

The fluid dynamics of biological fluids under the influence of high magnetic fields strength and its possible medical applications, was first investigated by Haik et al., [1]. The applications in medical sciences include cell separation, magnetic tracers and magnetic drug targeting. Blood is a biomagnetic fluid that can be affected by magnetic field such as deviation in blood flow [2]. Blood is magnetic due to the presence of Haemoglobin, a form of iron oxide [3]. Haemoglobin is a protein responsible for oxygen and carbon dioxide transportation throughout the body and constitutes almost one third of the red blood cells. This protein influences the orientation of normal red blood

\footnotetext{
* Corresponding author.

E-mail address: zuhaila@utm.my (Zuhaila Ismail)
}

https://doi.org/10.37934/cfdl.12.4.4353 
cells in magnetic field [4]. The magnetic property of blood changes depending on its oxygenation state; diamagnetic when oxygenated and paramagnetic when deoxygenated.

Tzirtzilakis [3,5] developed numerical models to investigate the interaction between blood flow and magnetic field in a rectangular channel using the principles of ferrohydrodynamics (FHD) [6]. With the presence of spatially varying magnetic field, flow pattern changes considerably especially in the vicinity of the magnetic source. Recently, Tzirtzilakis and Xenos [7] investigated the influence of a steady localized magnetic field on biomagnetic fluid in a lid driven cavity using finite volume method. The results showed formation of local vortices near the magnetic source. Abdullah et al., [8] carry out numerical study of biomanetic fluid dynamics (BFD) in a lid driven cavity with an oscillating lid using finite difference method (FDM) and study the influence of Reynolds number, magnetic number and oscillation frequency on the flow field. With an increase in the magnetic field intensity, the vortices within the cavity were observed to become increasingly large, enhancing the mixing process. The finite element method (FEM) has also been successfully used to solve BFD problems such as biomagnetic micropolar flow and magnetohydrodynamic flow in a square cavity [9-11].

As far as author's knowledge, very few studies concerning BFD in lid driven cavity using finite element method has been made. Considering the advantages of finite element method in solving problems involving complex geometry and/or boundary conditions, the main purpose of this paper is to simulate biomagnetic fluid flow in a lid driven cavity using both FEM and FDM. This serves as a fundamental work to investigate any stability issue in both methods especially in the presence of highly nonlinear body force. The results show that the FEM has issue resolving the steep magnetic field gradient and requires some stabilization while FDM is free from stability issue. Result concerning the resulting flow pattern is presented and discussed.

\section{Mathematical Formulation}

Mathematical model proposed in Tzirtzilakis [5] is adopted in this study. The problem is considered as two-dimensional while the flow is assumed to be steady and laminar. The biofluid is considered as Newtonian and non-conducting. It is also assumed that the temperature changes are negligible (isothermal) and magnetic field is sufficient enough to saturate the biofluid. The rotational forces acting on the erythrocytes, when entering and exiting the magnetic field are discarded (equilibrium magnetization).

Based on the above assumptions, the governing equations for this problem are similar to those derive by Haik et al., [1] and Rosensweig [12] which follows the principle of FHD. The equations are given by,

Momentum equation in $x$-direction:

$$
\bar{\rho}\left(\bar{u} \frac{\partial \bar{u}}{\partial \bar{x}}+\bar{v} \frac{\partial \bar{u}}{\partial \bar{y}}\right)=-\frac{\partial \bar{p}}{\partial \bar{x}}+\bar{\mu}_{0} \bar{M} \frac{\partial \bar{H}}{\partial \bar{x}}+\bar{\mu}\left(\frac{\partial^{2} \bar{u}}{\partial \bar{x}^{2}}+\frac{\partial^{2} \bar{u}}{\partial \bar{y}^{2}}\right)
$$

Momentum equation in $y$-direction:

$$
\bar{\rho}\left(\bar{u} \frac{\partial \bar{v}}{\partial \bar{x}}+\bar{v} \frac{\partial \bar{v}}{\partial \bar{y}}\right)=-\frac{\partial \bar{p}}{\partial \bar{y}}+\bar{\mu}_{0} \bar{M} \frac{\partial \bar{H}}{\partial \bar{y}}+\bar{\mu}\left(\frac{\partial^{2} \bar{v}}{\partial \bar{x}^{2}}+\frac{\partial^{2} \bar{v}}{\partial \bar{y}^{2}}\right)
$$

Continuity equation: 
$\frac{\partial \bar{u}}{\partial \bar{x}}+\frac{\partial \bar{v}}{\partial \bar{y}}=0$

A simple two-dimensional rectangular domain is adopted. The top wall is moving with a constant velocity, $\bar{u}_{r}$ along the $x$-direction while no-slip boundary condition is imposed on the rest of the walls. Pressure is applied at a single point on the bottom left corner of the cavity. The boundary conditions for the problem is summarized as follows

Lower wall $\quad \bar{u}(\bar{x}, 0)=0, \quad \bar{v}(\bar{x}, 0)=0$

Upper wall $\quad \bar{u}(\bar{x}, \bar{L})=\bar{u}_{r}, \quad \bar{v}(\bar{x}, \bar{L})=0$

Left wall $\quad \bar{u}(0, \bar{y})=0, \quad \bar{v}(0, \bar{y})=0$

Right wall $\quad \bar{u}(\bar{L}, \bar{y})=0, \quad \bar{v}(\bar{L}, \bar{y})=0$

In the above dimensional equations, $\bar{u}$ and $\bar{v}$ are the velocity components, $\bar{p}$ is the pressure, $\bar{\rho}$ is the biomagnetic fluid density, $\bar{\sigma}$ is the electrical conductivity, $\bar{\mu}$ is the dynamic viscosity and $\bar{\mu}_{0}$ is the magnetic permeability of the vacuum. $\bar{H}$ is the magnetic field intensity where the component is $\vec{H}=$ $\left(\bar{H}_{x}, \bar{H}_{y}\right)$, and $\bar{B}=\bar{\mu}_{0} \bar{H}$ is the magnetic induction where $\vec{B}=\left(\bar{B}_{x}, \bar{B}_{y}\right)=\bar{\mu}_{0}\left(\bar{H}_{x}, \bar{H}_{y}\right)$. The terms $\bar{\mu}_{0} \bar{M} \partial \bar{H} / \partial \bar{x}$ and $\bar{\mu}_{0} \bar{M} \partial \bar{H} / \partial \bar{y}$ from Eq. (1) and Eq. (2) respectively represents the magnetization force per unit mass which arise due to FHD.

Magnetization property described the behaviour of biofluid under influence of magnetic field (magnetized) [3]. According to the equilibrium magnetization, variation of magnetization, $\bar{M}$ with the magnetic intensity $\bar{H}$ can be expressed in linear relation as

$\bar{M}=\chi \bar{H}$

where $\chi$ is a constant called magnetic susceptibility. The components of the magnetic field intensity $\bar{H}_{x}$ and $\bar{H}_{y}$ along the $\bar{x}$ and $\bar{y}$ coordinates are

$\bar{H}_{x}=\frac{\gamma}{2 \pi} \frac{\bar{y}-\bar{b}}{(\bar{x}-\bar{a})^{2}+(\bar{y}-\bar{b})^{2}}$

$\bar{H}_{y}=-\frac{\gamma}{2 \pi} \frac{\bar{x}-\bar{a}}{(\bar{x}-\bar{a})^{2}+(\bar{y}-\bar{b})^{2}}$

where $(\bar{a}, \bar{b})$ is the point where the magnetic source is placed and $\gamma$ is the magnetic field strength at the point $(\bar{x}=\bar{a}, \bar{y}=\bar{b})$. The magnitude $\bar{H}$, of the magnetic field intensity, is given by

$\bar{H}=\left[\bar{H}_{x}{ }^{2}+\bar{H}_{y}{ }^{2}\right]^{\frac{1}{2}}=\frac{\gamma}{2 \pi} \frac{1}{\sqrt{(\bar{x}-\bar{a})^{2}+(\bar{y}-\bar{b})^{2}}}$

\section{Transformation of Equation}

Eqs. (1 - 4) and Eq. (8) are converted to non-dimensional form using the following dimensionless variables:

$x=\frac{\bar{x}}{\bar{L}}, \quad y=\frac{\bar{y}}{\bar{L}}, \quad u=\frac{\bar{u}}{\bar{u}_{r}}, \quad v=\frac{\bar{v}}{\bar{u}_{r}}, \quad p=\frac{\bar{p}}{\bar{\rho} \bar{u}_{r}^{2}}, \quad H_{x}=\frac{\bar{H}_{x}}{\bar{H}_{0}}, \quad H_{y}=\frac{\bar{H}_{y}}{\bar{H}_{0}}$ 
where $\bar{H}_{0}=\bar{H}(\bar{a}, 0)$. Substituting the dimensionless variables into Eqs. (1-4) and Eq. (8) resulted in the following set of equations:

$u \frac{\partial u}{\partial x}+v \frac{\partial u}{\partial y}=-\frac{\partial p}{\partial x}+M n_{F} H \frac{\partial H}{\partial x}+\frac{1}{R e}\left(\frac{\partial^{2} u}{\partial x^{2}}+\frac{\partial^{2} u}{\partial y^{2}}\right)$

$u \frac{\partial v}{\partial x}+v \frac{\partial v}{\partial y}=-\frac{\partial p}{\partial y}+M n_{F} H \frac{\partial H}{\partial y}+\frac{1}{R e}\left(\frac{\partial^{2} v}{\partial x^{2}}+\frac{\partial^{2} v}{\partial y^{2}}\right)$

$\frac{\partial u}{\partial x}+\frac{\partial v}{\partial y}=0$

The dimensionless form of the boundary conditions are given by:

Lower wall $\quad u(x, 0)=0, \quad v(x, 0)=0$

Upper wall $\quad u(x, 1)=1, \quad v(x, 1)=0$

Left wall $\quad u(0, y)=0, \quad v(0, y)=0$

Right wall $u(1, y)=0, v(1, y)=0$

The magnitude $H$ of the magnetic field intensity at the point $(a, b)$ is also derived by applying the relation in Eq. (6) and Eq. (7) and is given by

$H=\frac{|b|}{\sqrt{(x-a)^{2}+(y-b)^{2}}}$

The non-dimensional parameters are defined as:

$R e=\frac{\bar{L} \bar{\rho} \bar{u}_{r}}{\bar{\mu}}, \quad M n_{F}=\frac{\bar{\mu}_{0} \chi \bar{H}_{0}{ }^{2}}{\bar{\rho} \bar{u}_{r}{ }^{2}}$

where $R e$ is Reynolds number and $M n_{F}$ is the magnetic number arising due to FHD. The parameter for magnetic number $M n_{F}$ can also be written in the form of magnetic induction, $\overline{B_{o}}$ and the magnetization, $\overline{M_{o}}$ at $(a, 0)$ where

$M n_{F}=\frac{\overline{B_{O}} \overline{M_{O}}}{R e^{2}}$

When the magnetic number is zero, the problem reduces to common hydrodynamic flow of lid driven cavity.

\section{Numerical Method}

\subsection{Finite Difference Formulation}

The finite difference method used to solve Eq. (10) to Eq. (12) is based on the Marker and Cell method by Harlow and Welch [13] but highly simplified. This method is introduced as a numerical solution algorithm for transient fluid flows (SOLA) scheme [14], a type of projection and pressure correction method that has been used and extended to solve many fluid flow problems [15]. In this method, the staggered grid is used, hence the pressure $p$, velocity $u$ and $v$ are not located on the same grid. Using the staggered grid arrangement for the flow variables prevents the pressure 
oscillations that could occur if all the unknowns flow variables $p, u$, and $v$ are computed in the same grid.

To implement this scheme, the new time step velocity fields $u^{n+1}, v^{n+1}$ are to be calculated from Eq. (19) once the corresponding pressure is known. Therefore, the first step of this method is to calculate the new abbreviations $F$ and $G$ at time level $n$ as given below,

$$
\begin{aligned}
& F^{n}=u^{n}+\Delta t\left[-\left(u \frac{\partial u}{\partial x}+v \frac{\partial u}{\partial y}\right)+\frac{1}{R e}\left(\frac{\partial^{2} u}{\partial x^{2}}+\frac{\partial^{2} u}{\partial y^{2}}\right)+M n_{F} H \frac{\partial H}{\partial x}\right] \\
& G^{n}=v^{n}+\Delta t\left[-\left(u \frac{\partial v}{\partial x}+v \frac{\partial v}{\partial y}\right)+\frac{1}{R e}\left(\frac{\partial^{2} v}{\partial x^{2}}+\frac{\partial^{2} v}{\partial y^{2}}\right)+M n_{F} H \frac{\partial H}{\partial y}\right]
\end{aligned}
$$

where $\Delta t$ is the time step. The spatial derivatives on convection and diffusion terms are discretised using the finite difference central difference method, while the FHD terms are calculated analytically. A grid size of $150 \times 150$ is used and time step $\Delta t=0.001$. Once $F$ and $G$ at time level $n$, it will be used in the Poisson Pressure Equation given by

$\frac{\partial^{2} p^{n+1}}{\partial x^{2}}+\frac{\partial^{2} p^{n+1}}{\partial y^{2}}=\frac{1}{\Delta t}\left(\frac{\partial F^{n}}{\partial x}+\frac{\partial G^{n}}{\partial y}\right)$

The Poisson Pressure Equation is solved by using the preconditioned generalized minimum residual (GMRES) method. Other methods such as preconditioned conjugate gradient, and Successive Overrelaxation (SOR) has been tested as well and no difference is observed. The new velocity field $u^{n+1}$ and $v^{n+1}$ are then evaluated by the discretize momentum equations

$$
\begin{aligned}
& u^{n+1}=F^{n}-\Delta t \frac{\partial p^{n+1}}{\partial x} \\
& v^{n+1}=G^{n}-\Delta t \frac{\partial p^{n+1}}{\partial y}
\end{aligned}
$$

The above steps are iterated until steady state flow is achieved in which the tolerance is set to be $\left|u^{n+1}-u^{n}\right| \operatorname{AND}\left|v^{n+1}-v^{n}\right|<10^{-7}$.

\subsection{Finite Element Formulation}

The spatial discretization of the governing equations is performed using Galerkin weighted residual finite element method. Mixed formulation, in which the velocity and pressure are approximated using different shape functions, is adopted in this study. The Galerkin weighted residual method is applied by forcing the integral of the product between prescribed weight functions and the governing equations to zero. By subsequently taking integration by parts for the second order derivatives terms, the following set of equations are produced:

$$
\begin{aligned}
& \int_{A_{e}} N_{l}\left(u \frac{\partial N_{j} u_{j}}{\partial x}+v \frac{\partial N_{j} u_{j}}{\partial y}+\frac{\partial P_{k} p_{k}}{\partial x}\right) d A_{e}+\int_{A_{e}} \frac{1}{R e}\left(\frac{\partial N_{l}}{\partial x} \frac{\partial N_{j} u_{j}}{\partial x}+\frac{\partial N_{l}}{\partial y} \frac{\partial N_{j} u_{j}}{\partial y}\right) d A_{e}- \\
& \int_{A_{e}} N_{l} M n_{F} H \frac{\partial H}{\partial x} d A_{e}-B_{c x}=0 \\
& \int_{A_{e}} N_{l}\left(u \frac{\partial N_{j} v_{j}}{\partial x}+v \frac{\partial N_{j} v_{j}}{\partial y}+\frac{\partial P_{k} p_{k}}{\partial y}\right) d A_{e}+\int_{A_{e}} \frac{1}{R e}\left(\frac{\partial N_{l}}{\partial x} \frac{\partial N_{j} v_{j}}{\partial x}+\frac{\partial N_{l}}{\partial y} \frac{\partial N_{j} v_{j}}{\partial y}\right) d A_{e}- \\
& \int_{A_{e}} N_{l} M n_{F} H \frac{\partial H}{\partial y} d A_{e}-B_{c y}=0
\end{aligned}
$$


$\int_{A_{e}} P_{m}\left(\frac{\partial N_{j} u_{j}}{\partial x}+\frac{\partial N_{j} v_{j}}{\partial y}\right) d A_{e}=0$

In the above equation, $P$ and $N$ are linear and quadratic shape function respectively while $m, j, l$ are the index of the corresponding vectors. The right-hand terms in Eq. (20) and Eq. (21) are the boundary terms resulted from the integration by parts.

Eq. (20) to Eq. (22) can be written in a compact form as

$\left[\begin{array}{ccc}k_{11} & 0 & k_{13} \\ 0 & k_{22} & k_{23} \\ k_{31} & k_{32} & 0\end{array}\right]\left\{\begin{array}{l}u \\ v \\ p\end{array}\right\}=\left[\begin{array}{c}f_{u} \\ f_{v} \\ 0\end{array}\right]$

where

$k_{11}=\int_{A_{e}} N_{l} u \frac{\partial N_{j}}{\partial x}+\frac{1}{R e}\left(\frac{\partial N_{l}}{\partial x} \frac{\partial N_{j}}{\partial x}+\frac{\partial N_{l}}{\partial y} \frac{\partial N_{j}}{\partial y}\right) d A_{e}$

$k_{13}=\int_{A_{e}} N_{l} \frac{\partial P_{k}}{\partial x} d A_{e}$

$k_{22}=\int_{A_{e}} N_{l} v \frac{\partial N_{j}}{\partial x}+\frac{1}{R e}\left(\frac{\partial N_{l}}{\partial x} \frac{\partial N_{j}}{\partial x}+\frac{\partial N_{l}}{\partial y} \frac{\partial N_{j}}{\partial y}\right) d A_{e}$

$k_{23}=\int_{A_{e}} N_{l} \frac{\partial P_{k}}{\partial y} d A_{e}$

$k_{31}=\int_{A_{e}} P_{m} \frac{\partial N_{j}}{\partial x} d A_{e}$

$k_{32}=\int_{A_{e}} P_{m} \frac{\partial N_{j}}{\partial y} d A_{e}$

$f_{u}=N_{l} M n_{F} H \frac{\partial H}{\partial x}+\int_{A_{e}} \frac{1}{R e}\left(N_{l} \frac{\partial N_{j} v_{j}}{\partial x} n_{x}+N_{l} \frac{\partial N_{j} v_{j}}{\partial y} n_{y}\right) d S$

$f_{v}=N_{l} M n_{F} H \frac{\partial H}{\partial y}+\int_{A_{e}} \frac{1}{R e}\left(N_{l} \frac{\partial N_{j} v_{j}}{\partial x} n_{x}+N_{l} \frac{\partial N_{j} v_{j}}{\partial y} n_{y}\right) d S$

Eq. (23) is assembled into the global form before boundary condition from Eq. (13) are applied. Finally, the matrix system is solved using Gauss elimination technique with pivoting.

\section{Results and Discussion}

In this study, blood with density and dynamic viscosity of $\bar{\rho}=1050 \mathrm{kgm}^{-3}$ and $\bar{\mu}=3.2 \times$ $10^{-3} \mathrm{kgm}^{-1} \mathrm{~s}^{-1}$ respectively, is used as the fluid of interest. The computational domain consists of a square cavity with the side lengths of $0.05 \mathrm{~m}$. The top lid is moving with a horizontal velocity of $\bar{u}_{r}=$ $2.438 \times 10^{-2} \mathrm{~ms}^{-1}$ in the positive x-direction. The corresponds Reynolds number is $R e=400$, consistent with the parameter used in Tzirtzilakis and Xenos [7].

The source code for hydrodynamic problem without any magnetic source $\left(M n_{F}=0\right)$ is validated with an established lid driven cavity solution by Ghia et al., [16]. A comparison for the velocity in both $x$ and $y$ direction at the centre of the domain between the present numerical methods and Ghia et al., [16] for $R e=400$ is presented in Figure 1. It is clear from the figure that excellent agreement between the present methods and benchmark result is demonstrated.

With the application of magnetic source term at the bottom center of the domain, it is expected that similar result obtained by Tzirtzilakis and Xenos [7] would be observed for both FDM and FEM. Unfortunately, this is not the case. The FEM fail to predict the expected result. The streamline plot for cavity flow with magnetic field strength of $B_{0}=8 \mathrm{~T}$, corresponding to $M n_{F}=512.7$, using FEM for various grid size density is presented in Figure 2. Despite the disturbance of the streamline near the magnetic source, no vortices were observed even for increasing grid density. A detail 
investigation of the flow field for the FEM result is carried out by observing the velocity vector near the magnetic source. This is presented in Figure 3. From the figure, it can be clearly seen that the velocity vector does not behave entirely smooth, indicating some sort of numerical instability. This stability issue will be address next. FDM on the other hand does not suffer this problem and able to produce the desired result.

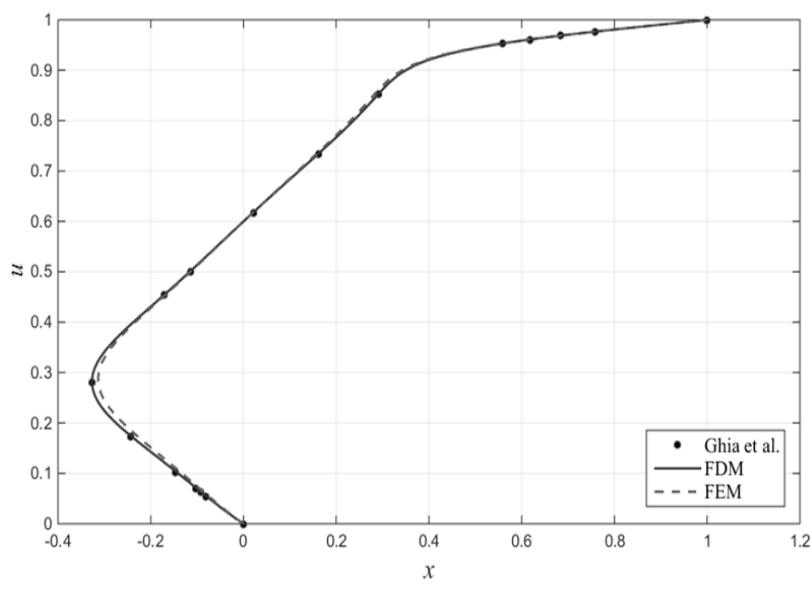

(a) $u$-center velocity

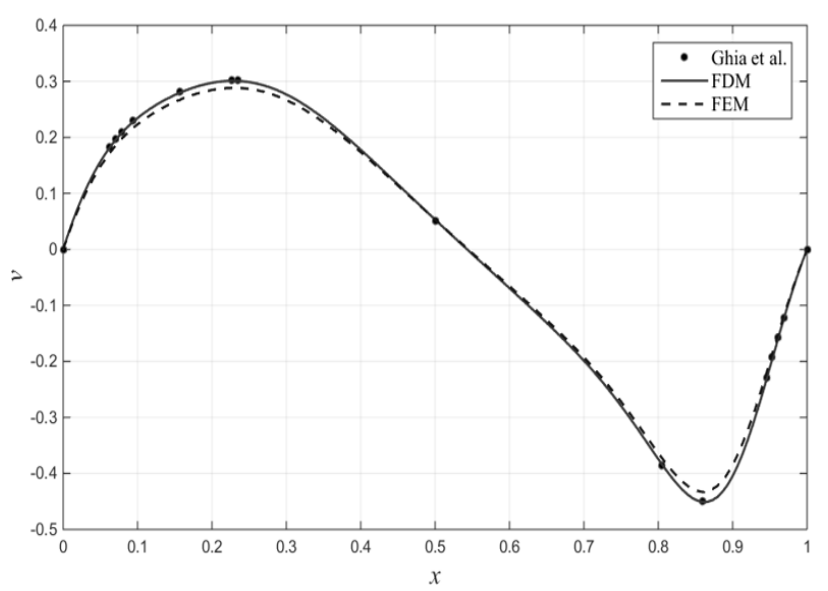

(b) $v$-center velocity

Fig. 1. Velocity at the center of the domain between present method and Ghia et al., [16] for $R e=400$

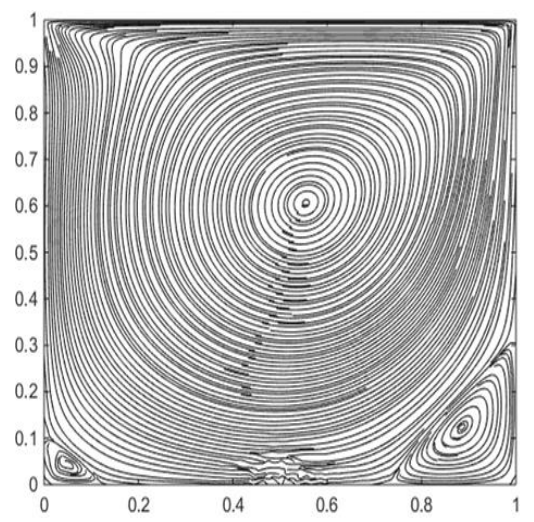

(a) $100 \times 100$

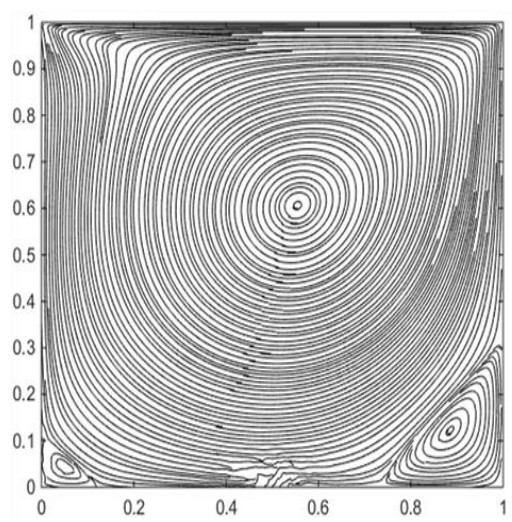

(b) $151 \times 151$

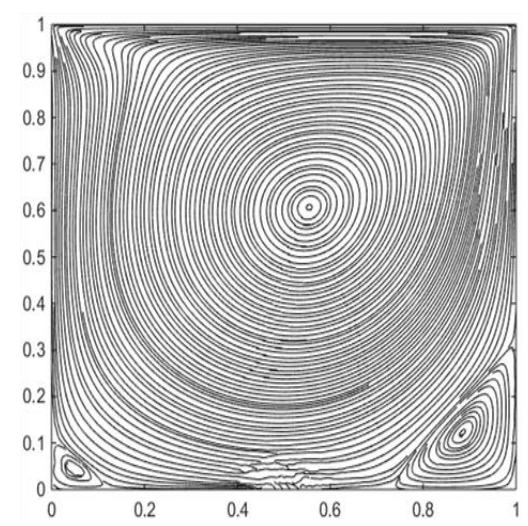

(c) $200 \times 200$

Fig. 2. Streamlines for lid driven cavity flow at $R e=400$ and $M n_{F}=512.70$ for difference grid size using FEM

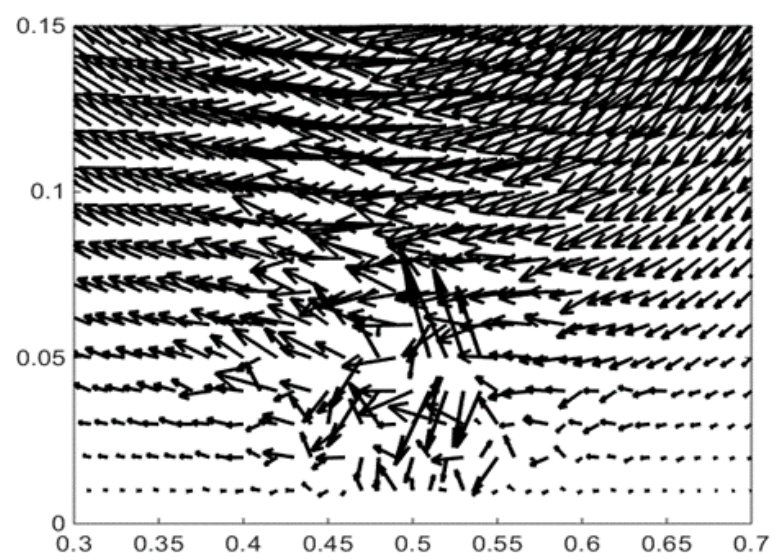

Fig. 3. Velocity vectors (near magnetic source) for lid driven cavity flow at $R e=400$ and $M n_{F}=$ 512.70 using FEM 
The source of numerical instability for the FEM result is believed to originate from the magnetic term in the momentum equation. Due to the nature of the magnetic field intensity, the magnetic field has a sharp gradient especially at the bottom center of the domain. Similarly, the derivatives of the magnetic field intensity, $H$, have a very sharp gradient near the source. These derivatives also have a large magnitude and could be another cause of numerical instability.

To mitigate this problem, a stabilization terms is added to the magnetic source term to dampen out of the sharp magnetic field gradient near the source. The stabilization term, $K$ is spatially varying in nature, with zero value at the magnetic source and rapidly takes the value of unity outward. The term has the following form

$K=1-\left[\frac{|c|}{\sqrt{(x-a)^{2}+(y-c)^{2}}}\right]$

where $a$ and $c$ are the constants that control the intensity of the term. The stabilization term is added to the momentum equations as follows

$$
\begin{aligned}
& u \frac{\partial u}{\partial x}+v \frac{\partial u}{\partial y}=-\frac{\partial p}{\partial x}+M n_{F} K H \frac{\partial H}{\partial x}+\frac{1}{R e}\left(\frac{\partial^{2} u}{\partial x^{2}}+\frac{\partial^{2} u}{\partial y^{2}}\right) \\
& u \frac{\partial v}{\partial x}+v \frac{\partial v}{\partial y}=-\frac{\partial p}{\partial y}+M n_{F} K H \frac{\partial H}{\partial y}+\frac{1}{R e}\left(\frac{\partial^{2} v}{\partial x^{2}}+\frac{\partial^{2} v}{\partial y^{2}}\right) \\
& \frac{\partial u}{\partial x}+\frac{\partial v}{\partial y}=0
\end{aligned}
$$

Figure 4 shows the results of the streamline for both FDM and FEM with and without the stabilization term. The results show with the application of the stabilization term, the correct result can be obtained, highlighting the success of the proposed solution.

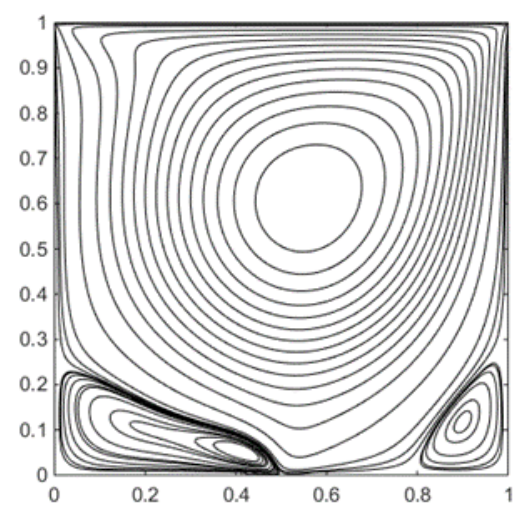

(a)

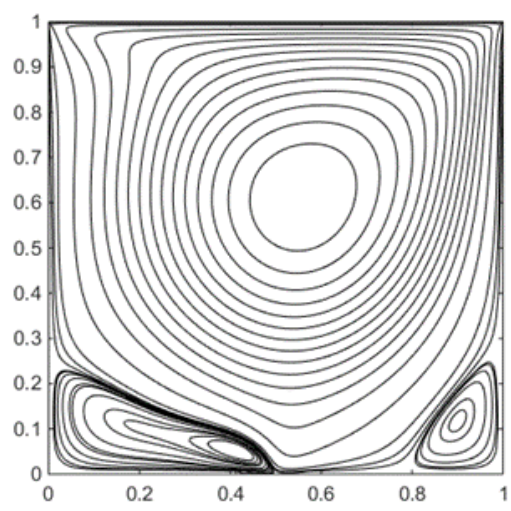

(b)

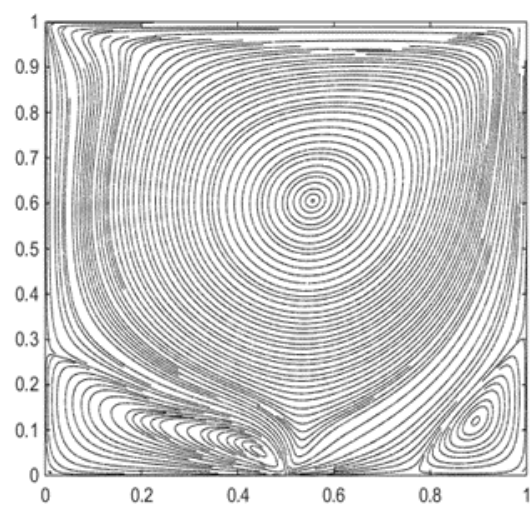

(c)

Fig. 4. Streamlines for $R e=400$ and $M n_{F}=512.70$ for (a) FDM without stabilization term (b) FDM with stabilization term (d) FEM with stabilization term. The constant $a=0.5$ and $c=0.0001$

With the treatment applied to the FEM, Figure 5 shows the streamline of BFD flow between present study and Tzirtzilakis and Xenos [7] for various magnetic field strength. For magnetic field strength of $B_{0}=0 \mathrm{~T}, 1 \mathrm{~T}, 4 \mathrm{~T}$ and $8 \mathrm{~T}$, the corresponding magnetic numbers are $M n_{F}=$ $0,64.09,256.35$ and 512.70 respectively. From the figure, it can be seen that the solutions show excellent agreement for all cases. 

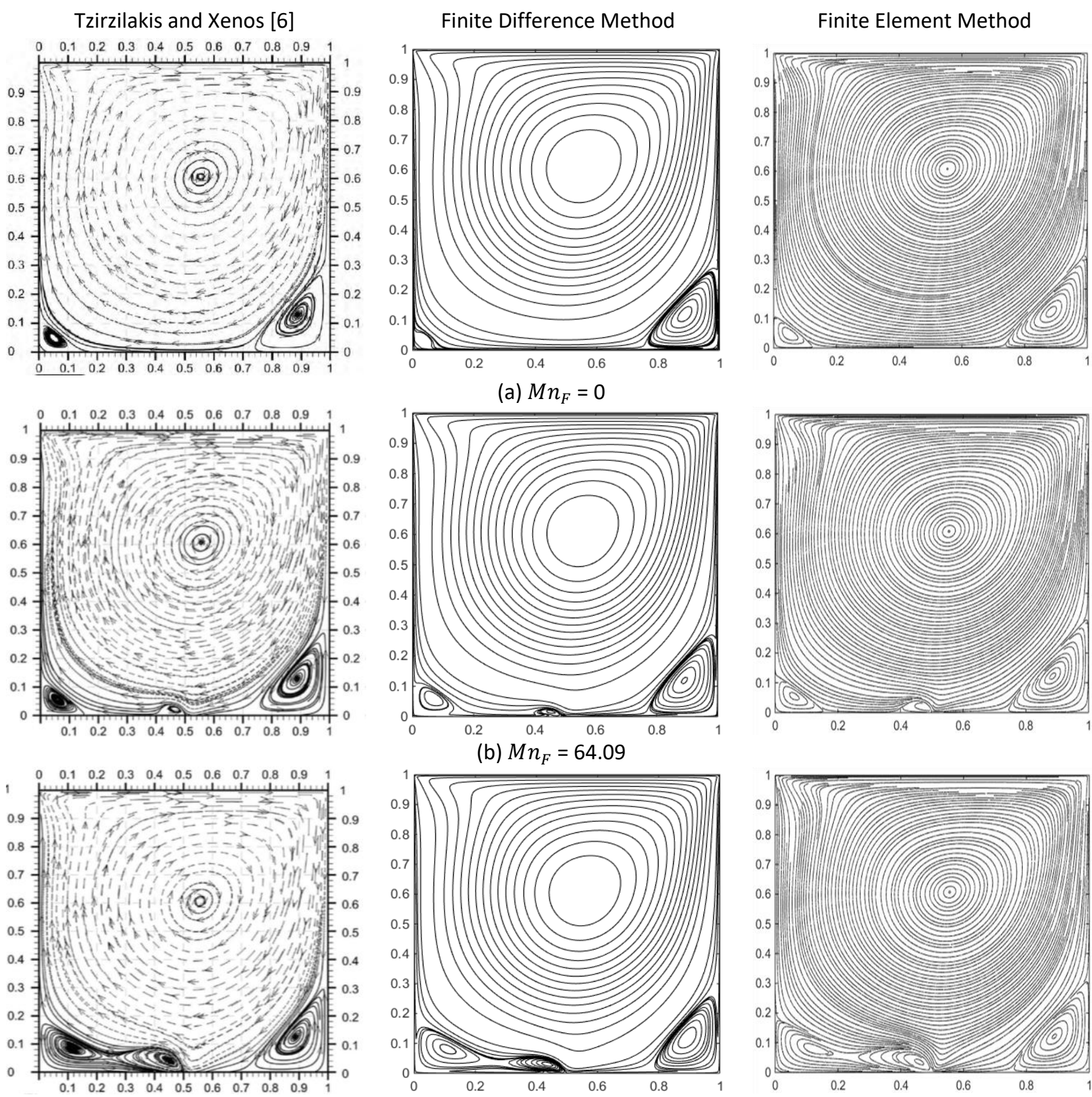

(c) $M n_{F}=256.35$
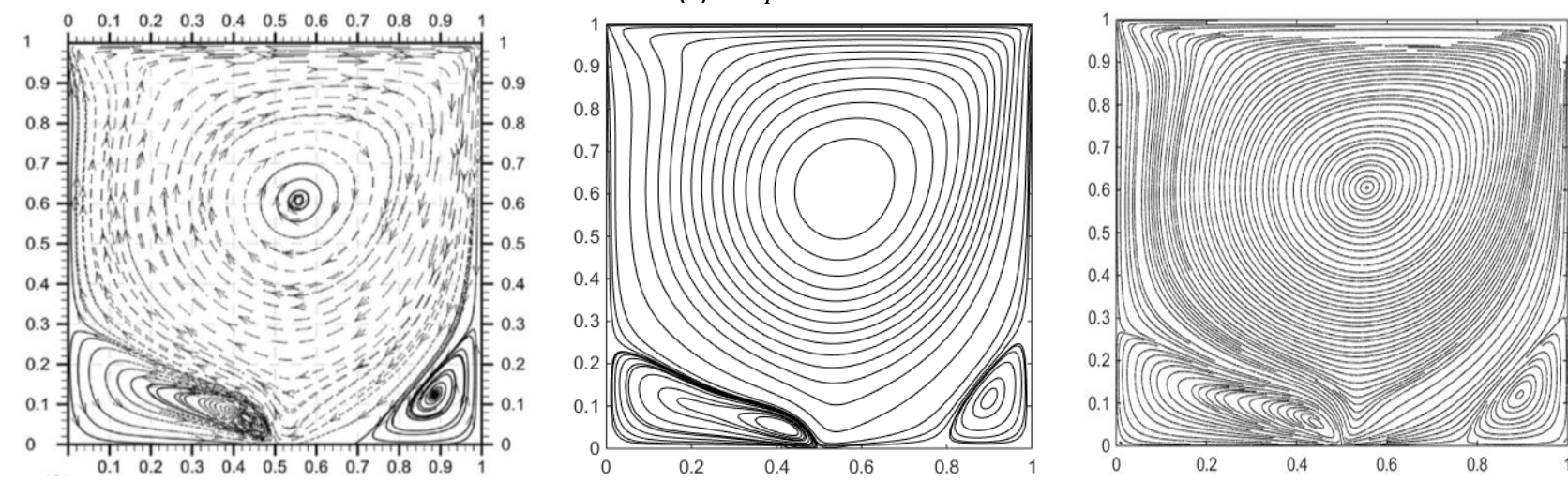

(d) $M n_{F}=512.70$

Fig. 5. Streamlines for $R e=400$ for various magnetic field strength between Tzirtzilakis and Xenos [7] (first column), finite difference method (second column) and finite element method (third column) 
For flow without magnetic field, only two vortices are present at the bottom left and right of the domain as shown in Figure 1(a). With an increase in the magnetic field strength, a pronounced vortex can be seen at the bottom center. For $B_{0}=1 \mathrm{~T}$, the vortex is barely noticeable. As the magnetic field strength increases $\left(B_{0}=4 \mathrm{~T}\right)$, the vortex at the center become increasingly larger and start entrapping the vortex at the bottom left corner. Finally, with the highest magnetic field strength of $B_{0}=8 \mathrm{~T}$, the recirculation pattern for the two vortices cannot be distinguished as they act as a single vortex. This vortex has a strong flow recirculation at magnetic field source.

\section{Conclusion}

The biomagnetic fluid flow in a lid driven cavity has been investigated numerically. The solution is obtained using both FDM and FEM. A stabilization term is introduced in the FEM formulation to mitigate stability issue relating to steep magnetic field gradient. Considering that FEM is excellent for problem involving complex geometry, the present result can be extended to model blood flow involving realistic domain subjected to spatially varying magnetic field.

\section{Acknowledgement}

The authors would like to acknowledge Ministry of Higher Education and Research Management Centre, Universiti Teknologi Malaysia for the financial support through vote numbers Q.J130000.2554.21H48 (UTMFR Grant) and R.J130000.7854.5F255 (FRGS Grant).

\section{References}

[1] Haik, Y., Pai, V., and Chen, C.J. "Biomagnetic fluid dynamics." Fluid dynamics at interfaces (1999): 439-452.

[2] Yadegari, Mehrdad, Mahdi Jahangiri, and Yadegari Mehrdad. "Simulation of non-Uniform Magnetic Field Impact on non-Newtonian and Pulsatile Blood Flow." Journal of Advanced Research in Fluid Mechanics and Thermal Sciences 57, no. 1 (2019): 86-99.

[3] Tzirtzilakis, E.E., Sakalis, V.D., Kafoussias, N.G. and Hatzikonstantinou, P.M. "Biomagnetic fluid flow in a 3D rectangular duct." International Journal for Numerical Methods in Fluids 44, no. 12 (2004): 1279-1298. https://doi.org/10.1002/fld.618

[4] Higashi, T., Yamagishi, A., Takeuchi, T., Kawaguchi, N., Sagawa, S., Onishi, S., and Date. M. "Orientation of erythrocytes in a strong static magnetic field." Blood 82, no. 4 (1993): 1328-1334.

https://doi.org/10.1182/blood.V82.4.1328.1328

[5] Tzirtzilakis, E.E. "A mathematical model for blood flow in magnetic field." Physics of fluids 17, no. 7 (2005): 077103. https://doi.org/10.1063/1.1978807

[6] Tzirtzilakis, E.E. "A simple numerical methodology for BFD problems using stream function vorticity formulation." Communications in Numerical Methods in Engineering 24, no. 8 (2008): 683-700. https://doi.org/10.1002/cnm.981

[7] Tzirtzilakis, E.E., and Xenos, M.A. "Biomagnetic fluid flow in a driven cavity." Meccanica 48, no. 1 (2013): $187-200$. https://doi.org/10.1007/s11012-012-9593-7

[8] Abdullah, A.A.A.A., Alnaqi, A., and Hossain, M.A. "Numerical Simulation of Biomagnetic Fluid Flow in an Oscillating Lid Driven Cavity." International Journal of Applied Engineering Research 11, no. 23 (2016): 11259-11265.

[9] Bhargava, R., Bég, O.A., Sharma, S., and Zueco, J. "Finite element study of nonlinear two-dimensional deoxygenated biomagnetic micropolar flow." Communications in Nonlinear Science and Numerical Simulation 15, no. 5 (2010): 1210-1223. https://doi.org/10.1016/i.cnsns.2009.05.049

[10] Aydın, S.H., Nesliturk, A.l., and Tezer-Sezgin, M. "Two-level finite element method with a stabilizing subgrid for the incompressible MHD equations." International journal for numerical methods in fluids 62, no. 2 (2010): 188-210. https://doi.org/10.1002/fld.2019

[11] Marioni, L., Bay, F., and Hachem, E. "Numerical stability analysis and flow simulation of lid-driven cavity subjected to high magnetic field." Physics of fluids 28, no. 5 (2016): 057102. https://doi.org/10.1063/1.4948433

[12] Rosensweig, R.E. "Magnetic fluids." Annual review of fluid mechanics 19, no. 1 (1987): 437-461. 
[13] Harlow, F.H and Welch, J.E. "Numerical Calculation of Time-Dependent Viscous Incompressible Flow of Fluid with Free Surface." The physics of fluids 8, no. 12 (1965): 2182-89. https://doi.org/10.1063/1.1761178

[14] Hirt, C.W., Nicholas, B.D., and Romero, N.C. "A Numerical Solution Algorithm for Transient Fluid Flow." Los Alamos Scientific Laboratory Report (1975).

[15] Chorin, A.J. "Numerical Solution of the Navier-Stokes Equations." Mathematics of computation 22, no. 104 (1968):745-62. https://doi.org/10.1090/S0025-5718-1968-0242392-2

[16] Ghia, U., Ghia, K.N., and Shin, C.T. "High-Re solutions for incompressible flow using the Navier-Stokes equations and a multigrid method." Journal of computational physics 48, no. 3 (1982): 387-411.

https://doi.org/10.1016/0021-9991(82)90058-4 